\title{
A new Centronuclear Myopathy phenotype due to a novel Dynamin 2 mutation
}

Marc Bitoun $(\mathrm{PhD})^{1,2}$, Jorge A. Bevilacqua $(\mathrm{MD}, \mathrm{PhD})^{1,3}$, Bruno Eymard (MD, $\left.\mathrm{PhD}\right)^{4}$, Bernard Prudhon $(\mathrm{MSc})^{1,2}$, Michel Fardeau (MD) ${ }^{5,6}$, Pascale Guicheney $(\mathrm{PhD})^{1,2,5}$, Norma B. Romero (MD, PhD) $)^{1,2,5}$

1. Inserm, U582, Institut de Myologie, Paris, (F-75013), France.

2. UPMC Univ Paris 06, UMR_S582, IFR14, Paris, (F-75013), France.

3. Departamento de Neurología y Neurocirugía, HCUCH and Instituto de Ciencias Biomédicas Universidad de Chile, Santiago, Chile.

4. Centre de Référence de Pathologie Neuromusculaire Paris-Est, Groupe Hospitalier Pitié-Salpêtrière, Paris, France.

5. AP-HP, Groupe Hospitalier Pitié-Salpêtrière, Paris, (F-75013), France.

6. Association Institut de Myologie (AIM), Groupe Hospitalier Pitié-Salpêtrière, Paris, F-75013, France

Disclosure: The authors report no conflicts of interest.

Article: 749 words

Title: 72 characters

Search terms: Muscle disease [ 185 ], Clinical neurology examination [ 16 ], CT [ 119]

Corresponding Author:

Dr. Marc Bitoun, Inserm U582, Institut de Myologie, Groupe Hospitalier PitiéSalpêtrière, 75013, Paris, France. 
E-mail: m.bitoun@institut-myologie.org

Tel : 33 (0) 1.42.16.57.19

Fax: $33(0)$ 1.42.16.57.00

\section{Acknowledgements.}

We thank the Institut National de la Santé et de la Recherche Médicale (Inserm), the Association Française contre les Myopathies (AFM), and the Programme of Collaboration ECOS-SECyT ( ${ }^{\circ}$ A02S02) for financial supports, and Dr N Clarke for helpful advices. Jorge A. Bevilacqua was supported by the Programme Alban, the European Union Programme of High Level Scholarships for Latin America, scholarship No.E04E028343CL. 
Autosomal dominant Centronuclear Myopathy $(\mathrm{CNM})$ is a rare congenital myopathy mostly characterized by delayed motor milestones, slowly progressive muscle weakness and bilateral ptosis ${ }^{1}$. Mutations in the DNM2 gene encoding dynamin 2 (DNM2), a large GTPase involved in membrane trafficking, have been identified in $\mathrm{CNM}^{2,3}$. Mutations in the middle domain of the protein are mostly associated with the slowly progressive mild late-onset $\mathrm{CNM}^{2}$, while mutations in the C-terminal part of the Pleckstrin homology $(\mathrm{PH})$ domain cause a more severe neonatal phenotype ${ }^{3}$. In addition, mutations in the $\mathrm{N}$-terminal part of $\mathrm{PH}$ domain have been reported in intermediate and axonal Charcot-Marie-Tooth disease $(\mathrm{CMT})^{4,5,6}$. Here, we report a novel DNM2 CNM-mutation in the "CMT-region”. Among the DNM2-related CNM, the phenotype appears intermediate, with an onset at the end of the first decade and a more rapid progression relative to the mild late-onset DNM2-CNM.

\section{Case report.}

The patient is a 34-year-old woman from a non consanguineous family from central Africa without history of neuromuscular disorders. She had a normal mental and motor postnatal development with independent ambulation acquired at 13 months. Symptoms started at the age of 7 years with difficulty to walk and run. At 10 years, she had facial weakness, bilateral ptosis and a marked weakness in paraspinal, upper and lower limb muscles. Motor nerve conduction velocities of the left common peroneal $(49.4 \mathrm{~m} / \mathrm{s})$ and ulnar nerves $(53.5 \mathrm{~m} / \mathrm{s})$, and latencies were normal for age. Electromyography (EMG) revealed a myopathic pattern without neuropathic signs.

From the ages of 10 to 30 years, axial and limb muscle strength deteriorated continuously, but with a more pronounced aggravation between 11 and 15 years. She 
developed a severe ophthalmoparesis and progressive difficulty climbing stairs, frequent falls and dysphagia. At age 30 years, her maximum distance for walking was 20 meters and muscle CT scan assessment showed a diffuse involvement of limb muscles (Figure 1B-F). The posterior compartment of the forearms and thighs were relatively more affected than anterior compartments. In the legs, both anterior and posterior compartments were severely affected but the posterior compartment showed more marked abnormalities. At the last examination (34 years), she had severe arm weakness (shoulder antepulsion limited to $50^{\circ}$ and abduction to $40^{\circ}$ ), had recurrent back pain and required a wheelchair. Deep tendon reflexes were abolished in the 4 limbs and EMG showed a myopathic pattern without neuropathic signs. The vital capacity was $51 \%$ of the expected value at 12 years, $58 \%$ at 23 years, $67 \%$ at 30 years and $45 \%$ at 34 years, consistent with a restrictive respiratory syndrome.

Electrocardiogram and echocardiogram were normal. Serum creatine kinase levels and leukocyte count were in the normal range.

Deltoid muscle biopsy at the age of 10 years revealed nuclear centralization, predominance and hypotrophy of type 1 fibers and radial arrangement of sarcoplasmic strands (Figure 1G-I). Moderate endomysial fibrosis was observed, without evidence of necrosis or regeneration.

A DNA sample was extracted from blood and the DNM2 gene was sequenced. We identified a heterozygous mutation in exon 15. The mutation (c.G1678A) induces the change of the highly conserved glutamate 560 to lysine (p.E560K) located in the PH domain (Figure 1A).

\section{Discussion.}


We report a novel DNM2 mutation associated with CNM with a topography of muscle involvement similar to the already reported DNM2-CNM patients ${ }^{7}$, but with an unusual progressive course. From the ages of 10 to 15 years, she developed severe, generalized muscle weakness and required a wheelchair from age 30 years-old. Among the DNM2-CNM patients reported until now, the association of a stable restrictive respiratory syndrome with a relatively rapid evolution, compared to the mild late-onset CNM in which loss of ambulation mostly occurs after the $5^{\text {th }}$ decade, is unique. Consequently, our results enlarge the clinical spectrum of the DNM2-CNM which includes the severe neonatal and the slow progressive adult mild forms, and also intermediate CNM between these two phenotypes.

The p.E560K mutation is positioned in the region of the $\mathrm{PH}$ domain in which all the CMT-mutations were identified ${ }^{4,5,6}$. In particular, the p.K559del mutation was showed to cause axonal CMT in a patient with nerve conduction anomalies typical of axonal CMT and without evidence of muscular involvement on EMG and muscle biopsy ${ }^{6}$. Inversely, in the patient reported here, nerve conduction studies were normal at age 10 years, EMG revealed only a myopathic pattern at 10 and 34 years and muscle biopsy displayed the characteristic features of CNM. Therefore, the N-terminal part of the $\mathrm{PH}$ domain is not specifically linked to CMT but the cause of the tissue-specific phenotype remains to be determined.

\section{References.}

1. Jeannet PY, Bassez G, Eymard B, et al. Clinical and histologic findings in autosomal centronuclear myopathy. Neurology 2004;62:1484-1490. 
2. Bitoun M, Maugenre S, Jeannet $\mathrm{P}$, et al. Mutations in dynamin 2 cause dominant Centronuclear Myopathy. Nat Genet 2005;37:1207-1209.

3. Bitoun M, Bevilacqua JA, Prudhon B, et al. Dynamin 2 mutations cause sporadic centronuclear myopathy with neonatal onset. Ann Neurol 2007;62:666-670.

4. Züchner S, Noureddine M, Kennerson M, et al. Mutations in the pleckstrin homology domain of dynamin 2 cause dominant intermediate Charcot-Marie-Tooth disease. Nat Genet 2005;37:289-294.

5. Fabrizi GM, Ferrarini M, Cavallaro T, et al. Two novel mutations in dynamin-2 cause axonal Charcot-Marie-Tooth disease. Neurology 2007;69:291-295.

6. Bitoun M, Stojkovic T, Prudhon B, et al. A novel mutation in the dynamin 2 gene in a Charcot-Marie-Tooth type 2 patient: clinical and pathological findings. Neuromuscul Disord 2008;18:334-338.

7. Fischer D, Herasse M, Bitoun M, et al. Characterization of the muscle involvement in dynamin 2 related centronuclear myopathy. Brain 2006;129:1463-1469.

\section{Legends.}

Figure 1: Summary of mutated DNM2 amino-acids in human diseases and muscle features from the patient. 
A. DNM2 is composed of a GTPase domain (GTPase), a middle domain (Middle), a Pleckstrin homology (PH) domain, a GTPase effector domain (GED) and a Proline rich domain (PRD). CNM mutations (amino-acids indicated in bold case) are located in the middle domain and in the C-terminal part of the PH domain. CMT mutations are located in the N-terminal part of the PH domain. The novel DNM2-CNM (boxed) mutation p.E560K was identified in the protein region involved in CMT. The aminoacids were numbered according to the isoform 1 (Accession number: NP_001005360) which includes the four amino-acids GEIL at positions 516-519. B-F: Computer tomography scans of upper and lower limb muscles of patient at 28 years of age. Axial scanning sections corresponding to: $\mathbf{B}=$ arm, middle plane; $\mathbf{C}=$ forearm; $\mathbf{D}=$ pelvic girdle muscles; $\mathbf{E}=$ tight, middle plane and $\mathbf{F}=$ legs, middle plane. In $\mathrm{B}$, muscles of the arm are relatively less affected than the other muscular groups. Posterior compartments of the forearms (C) and thighs (E) are relatively more affected than anterior compartments at same level. Signal in proximal lower limb muscles is hypointense (D). At the level of the legs (F), both anterior and posterior compartments are affected. G-I: Deltoid muscle biopsy performed at the age of 10 years that shows characteristic CNM histopathological findings. Representative fields show central nuclei in $37 \%$ of fibers (G: Hematein-Eosin staining), radial distribution of sarcoplasmic strands in $22 \%$ of fibers (H: Nicotinamide Adenine DinucleotideTetrazolium Reductase staining) and predominance and hypotrophy of type 1 fibers (I: ATPase preincubated at $\mathrm{pH} 9.4$ staining). Type 1 fibers represent $98 \%$ of fibers. 

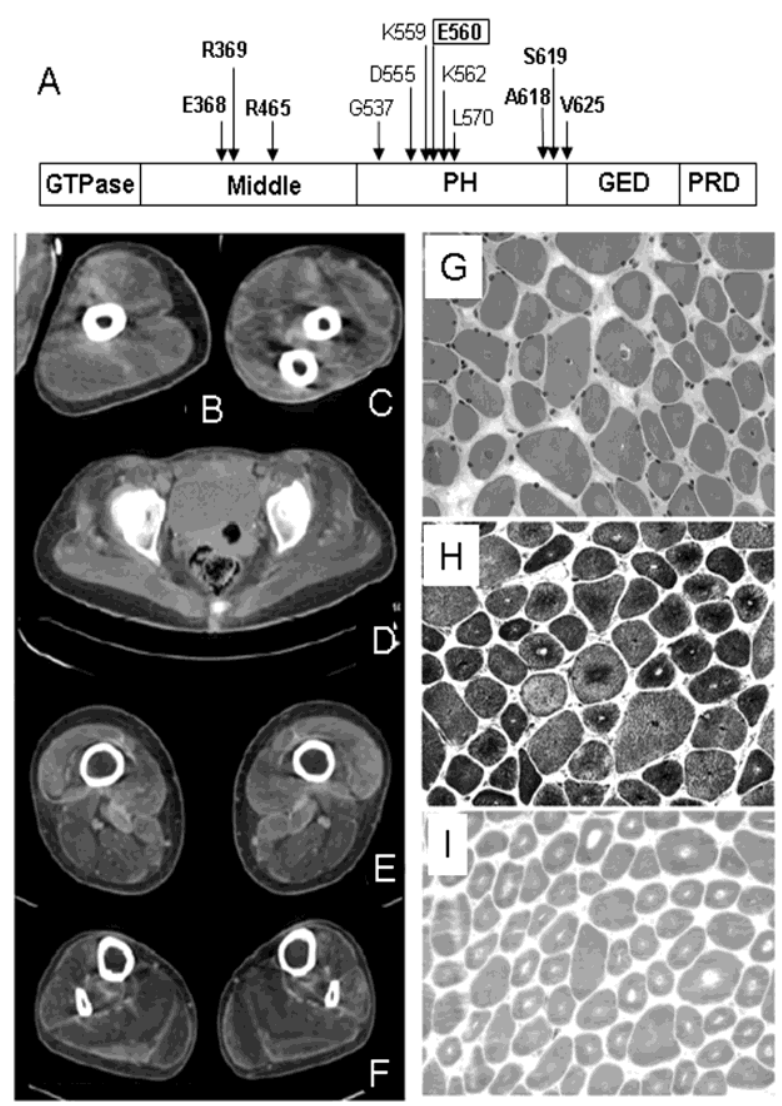

Figure 1 